\title{
$E V L$ (Ena/VASP-like) expression is up-regulated in human breast cancer and its relative expression level is correlated with clinical stages
}

\author{
LI-DE HU, HUA-FEI ZOU, SHU-XUAN ZHAN and KAI-MING CAO \\ State Key Laboratory of Genetic Engineering, Department of Biochemistry, \\ School of Life Sciences, Fudan University, Shanghai 200433, P.R. China
}

Received September 6, 2007; Accepted October 24, 2007

\begin{abstract}
EVL belongs to the Ena/VASP (Enabled/ vasodilator-stimulated phosphoprotein) family of proteins [Mena (mammalian Ena), VASP and EVL], which have a range of roles in regulating the actin cytoskeleton. Growing evidence suggests that Mena and VASP are involved in carcinogenesis, though little is known about the significance of EVL's function in cancer. This study examined the expression levels of $E V L$ mRNA in paired breast cancer specimens using semi-quantitative and real-time RT-PCR. In a comparison between matched breast tumors and normal tissues, a significant increase in the level of $E V L$ mRNA was found in 23 of the $35(65.7 \%)$ tumors $(\mathrm{P}=0.032)$. Patients in the advanced stages more frequently exhibited an elevated $E V L$ expression in tumor tissues. The EVL mRNA relative expression level significantly correlated with clinical stages $(\mathrm{P}=0.021)$. To begin elucidating the mechanism, we measured the ability of EVL to transform NIH3T3 cells and regulate the motility of MCF-7 cells in vitro by focus formation and modified Boyden chamber assays. The results indicated that overexpression of EVL was insufficient to transform NIH3T3 cells, although the motility of EVL transfected MCF-7 cells was markedly promoted. Collectively, EVL expression level was higher in breast tumors compared to normal tissues and its up-regulation was positively associated with the clinical stages of breast cancer. Additionally, EVL may be implicated in invasion and/or metastasis of human breast cancer.
\end{abstract}

\section{Introduction}

Breast cancer is one of the most common malignancies diagnosed among women (1). During the past few decades, the incidence rate of breast cancer has increased markedly in

Correspondence to: Professor Kai-Ming Cao, State Key Laboratory of Genetic Engineering, Department of Biochemistry, School of Life Sciences, Fudan University, Shanghai 200433, P.R. China

E-mail: kmcao@fudan.edu.cn

Key words: Enabled/vasodilator-stimulated phosphoprotein-like, breast cancer, clinical stage, quantitative real-time RT-PCR, cell migration
China, especially in metropolitan areas $(2,3)$. Thus, the identification of tumor-specific genes and the definition of their roles in breast cancer development will help to elucidate the molecular mechanisms of breast cancer and develop protocols for early clinical diagnosis and therapy.

The actin cytoskeleton engages in many cellular functions including the regulation of cell shape, growth, adherence and motility, which have escaped normal control mechanisms during malignant transformation and metastasis. Emerging data have suggested that the progression of breast cancer is accompanied by a de-regulation of actin cytoskeleton regulatory proteins $(4,5)$. Ena/VASP (Enabled/vasodilatorstimulated phosphoprotein) family proteins are involved in cell motility, adhesion and many other actin-dependent processes. Mammalian Ena/VASP proteins (Mena, VASP and EVL) have conserved domain organization and exhibited an extensive functional overlap $(6,7)$. Successive studies by $\mathrm{Di}$ Modugno et al showed that hMena (human Mena) was overexpressed in $>70 \%$ of primary breast cancers (8) and in benign breast lesions with a high risk of transformation (9). Up-regulated rat Mena was also detected in invasive mammary tumor cells (10). It was reported that lung adenocarcinomas show greater VASP expression than normal epithelium (11). $E V L$ was originally identified by its significant sequence similar to Ena and VASP and was involved in actin-based motility and localized to focal adhesions and the leading edge of lamellipodia in fibroblasts $(12,13)$.

To date, no studies have examined EVL expression in breast cancer, though previous studies suggested that Ena/ VASP proteins might be involved in carcinogenesis $(8-11,14)$. In order to understand whether an altered expression level of $E V L$ was involved in the progression of breast cancer, the mRNA expression levels of EVL in 35 paired breast cancer specimens were evaluated by semi-quantitative and real-time RT-PCR and the relative EVL expression levels were correlated with clinicopathological factors. Additionally, the transforming ability of EVL was assessed with a classical fibroblast assay and the role of EVL in regulating MCF-7 cell migration was examined by a modified Boyden chamber assay.

\section{Materials and methods}

Patients and tissue samples. Thirty-five patients with breast cancer who underwent surgery at Xinhua Hospital (Shanghai, 
P.R. China) from October 2006 to April 2007 were enrolled in this study. Fresh surgical tumors and corresponding normal tissue specimens were grossly dissected to maximize the removal of adipose tissues, snap-frozen in liquid nitrogen and stored at $-70^{\circ} \mathrm{C}$ until further processing. Histopathological analysis confirmed the surgical differentiation of malignant and surrounding normal tissues. Each tumor was categorized according to the AJCC staging system (15) and clinical data obtained from the medical records are displayed in Table I. Each study was conducted in accordance with the tenets of the Declaration of Helsinki.

RNA extraction and reverse transcription. Total RNA was extracted from 35 paired normal and tumor breast tissue specimens using Trizol ${ }^{\circledR}$ reagent (Invitrogen ${ }^{\mathrm{TM}}$ ). cDNA synthesis (every material purchased from Promega) was performed with an oligo-(dT) $)_{18}$ primer and M-MLV Reverse Transcriptase in the presence of RNase inhibitor following the manufacturer's protocol. Before being reverse transcribed, the RNA was treated with RQ1 RNase-free DNase to remove genomic DNA contamination. The reverse transcription of each specimen was performed three times independently and PCR assays were performed separately on the products.

Semi-quantitative PCR. The forward (5'-CAATGCTGTTTG CCCTGAA-3') and reverse (5'-GTTGGCATCGGACTTT GAG-3') primers for EVL (accession number NM_016337) were designed to generate a $458 \mathrm{bp}$ PCR product. $\beta$-actin (accession number NM_001101) primers were used concurrently as internal controls. The forward and reverse sequences were 5'-AGCGAGCATCCCCCAAAGTT-3' and 5'-GGGCACGAAGGCTCATCATT-3', respectively, which obtained a 285 bp PCR product. A prior PCR optimization was performed to ensure that each reaction was terminated during the exponential phase of the amplification. The PCR amplification was performed with Takara $\mathrm{Taq}^{\mathrm{TM}}$ for 1 cycle at $94^{\circ} \mathrm{C}$ for $5 \mathrm{~min}$ followed by 23 and 26 cycles for $\beta$-actin and $E V L$, respectively, at $94^{\circ} \mathrm{C}$ for $30 \mathrm{sec}, 58.5^{\circ} \mathrm{C}$ for $30 \mathrm{sec}$ and $72^{\circ} \mathrm{C}$ for $30 \mathrm{sec}$. The PCR products were separated on $1.5 \%$ agarose gels with ethidium bromide for visualization. Each test was repeated three times independently.

Quantitative real-time PCR. The Bio-Rad iCycler $\mathrm{iQ}^{\mathrm{TM}}$ system was employed to quantify the expression levels of $E V L$ and $\beta$-actin in the breast tissue. The specific primers for $E V L$ and $\beta$-actin were designed and synthesized by Takara and the forward and reverse primer sequences and corresponding amplicon sizes were as follows: for $E V L, 5$ '-CACGACGAG AGCTCCATGTCA-3', 5'-TGGCCAGCAGTTTGTTCAT TTC-3', $187 \mathrm{bp}$; for $\beta$-actin: 5'-TGGCACCCAGCACAAT GAA-3', 5'-CTAAGTCATAGTCCGCCTAGAAGCA-3', $186 \mathrm{bp}$.

Optimal PCR conditions were established using a SYBR Premix Ex Taq kit (Takara) in accordance with the manufacturer's instructions. A reaction $(20 \mu \mathrm{l})$ consisting of $1.2 \mu \mathrm{l}$ cDNA (corresponding to $20 \mathrm{ng}$ RNA), $10 \mu \mathrm{l}$ of SYBR Premix Ex Taq and $1 \mu \mathrm{l}$ of each $4 \mu \mathrm{M}$ primer. PCR amplification was performed as follows: pre-denaturation for one cycle at $95^{\circ} \mathrm{C}$ for $10 \mathrm{sec}$ and 40 cycles at $95^{\circ} \mathrm{C}$ for $5 \mathrm{sec}$, $60^{\circ} \mathrm{C}$ for $20 \mathrm{sec}$ and $72^{\circ} \mathrm{C}$ for $10 \mathrm{sec}$; data acquisition was performed at the chain extension step. A melting curve analysis of the PCR products was performed between $65^{\circ} \mathrm{C}$ and $95^{\circ} \mathrm{C}$ at $0.05^{\circ} \mathrm{C} / \mathrm{sec}$ increments. To verify the melting curve results, randomly selected PCR products were separated on agarose gels, recovered and sequenced.

For each specimen, the amount of EVL and $\beta$-actin mRNA was calculated based on standard curves generated from corresponding PCR products, which was obtained from a patient-derived sample. The relative expression level was expressed as a ratio of the amount of EVL mRNA to $\beta$-actin mRNA. In each case, the fold difference of relative $E V L$ expression in tumor tissue versus normal tissue was calculated using the formula ratio tumor $_{\text {ratio }} /$ normal . Three independent experiments were performed and three-well repeats were measured for each sample. A non-template control and standards were included in each experiment.

Cell lines and plasmid constructs. The human breast cancer cell line MCF-7 and mouse fibroblast NIH3T3 were kindly provided by the Shanghai Institute of Materia Medica, Chinese Academy of Sciences. Cells were grown in DMEM medium $\left(\mathrm{Gibco}^{\mathrm{TM}}\right)$ with $10 \%$ fetal bovine serum (HyClone) and other required supplements at $37^{\circ} \mathrm{C}$ in a humidified atmosphere containing $5 \% \mathrm{CO}_{2}$.

The full-length EVL and $H$-Ras (accession number NM_005343) cDNA was amplified by RT-PCR from total RNA isolated from a tumor breast tissue and inserted, respectively, into the cloning vector pGEM $^{\circledR}-\mathrm{T}$ (Promega). The specific primers used were: for $E V L, 5$ '-CCCTGTGCTGCC ACTTTTCA-3' (forward) and 5'-GCTCCTGTGCGTGCTC TGGT-3' (reverse); for H-Ras, 5'-CTGAGGAGCGATGAC GGAATA-3' (forward) and 5'-CCTTCCTTGCTTCCGTC CTT-3' (reverse).

The coding regions of $E V L$ and $H$-Ras were PCR amplified from pGEM-T-EVL or pGEM-T-H-Ras and cloned, respectively, into the mammalian expression vector pcDNA4/myc-His C (Invitrogen). The primers used were: for EVL, 5'-GCGGATCCGCCACCATGGCCACAAG-3' (forward) and 5'-AGGCCGGCTCGAGACGTGGTGCTG ATC-3' (reverse); for $H$-Ras, 5'-GAGGATCCGCCACCAT GACGGAATA-3' (forward) and 5'-TTGTGCCTCGAGAG GAGAGCACACA-3' (reverse). The BamHI restriction sites and the Kozak sequences were designed into the forward primers. In the reverse primers, the stop codons were mutated to be a part of the XhoI restriction sites and the ORF (open reading frame) of $E V L$ and $H$-Ras was designed to be inframe with the $\mathrm{C}$-terminal myc-His tag. The coding sequence of EVL was also amplified with the primers: 5'-CTTCC AAGCTTCAGCCATGGCCACA-3' (forward) and 5'-CGA CGGATCCGCGCAGCGAG-3' (reverse). It was then introduced into the HindIII/BamHI site of pEGFP-C1 (Clontech) and was fused with the EGFP (enhanced green fluorescent protein) gene. The restriction sites of the primers are underlined and the Kozak sequences are shown in bold. Each construct was verified by DNA sequencing (Invitrogen).

Western blot analysis. NIH3T3 cells transfected with pcDNA4/myc-His C, pcDNA4/myc-His C-EVL and 
Table I. Correlation of the fold differences of the EVL relative expression levels with patient clinicopathological parameters and molecular marker data in paired breast cancer specimens.

\begin{tabular}{|c|c|c|c|c|}
\hline Feature & Number of patients $(\%)$ & Median $^{\mathrm{a}}$ & Mean $^{\mathrm{a}}$ & P-value \\
\hline Age (Median, 56 yrs; Range, 43-82 yrs) & & & & $0.195^{\mathrm{b}}$ \\
\hline$<$ Median & $17(48.6)$ & 1.30 & 2.42 & \\
\hline$\geq$ Median & $18(51.4)$ & 3.27 & 4.36 & \\
\hline Clinical stage & & & & $0.0210^{\mathrm{c}, \mathrm{d}}$ \\
\hline I & $010(28.6)$ & 0.945 & 2.44 & \\
\hline II & $422(62.8)$ & 1.86 & 2.56 & \\
\hline III & $53(8.60)$ & 13.80 & 13.00 & \\
\hline Tumor maximal diameter $(\mathrm{cm})$ & & & & $0.228^{c}$ \\
\hline$\leq 2$ & $15(42.8)$ & 1.88 & 3.06 & \\
\hline$>2$ and $\leq 5$ & $19(54.3)$ & 1.83 & 2.94 & \\
\hline$>5$ & $1(2.90)$ & 17.80 & 17.80 & \\
\hline Histological type & & & & $0.687^{\mathrm{b}}$ \\
\hline Ductal & $24(68.6)$ & 1.62 & 3.06 & \\
\hline Lobular/others & $11(31.4)$ & 2.51 & 4.20 & \\
\hline Lymph node status & & & & $0.172^{\mathrm{b}}$ \\
\hline Negative & $23(65.7)$ & 1.30 & 2.68 & \\
\hline Positive & $12(34.3)$ & 2.20 & 4.84 & \\
\hline ER status & & & & $0.987^{\mathrm{b}}$ \\
\hline Negative & $14(40.0)$ & 1.74 & 3.55 & \\
\hline Positive & $21(60.0)$ & 1.88 & 3.33 & \\
\hline PR status & & & & $0.301^{\mathrm{b}}$ \\
\hline Negative & $16(45.7)$ & 2.62 & 4.28 & \\
\hline Positive & $19(54.3)$ & 1.30 & 2.70 & \\
\hline
\end{tabular}

${ }^{a}$ For each variable, the median and mean of the fold differences of $E V L$ relative expression levels in paired tumor tissues versus normal tissues are reported. ${ }^{b}$ The Mann-Whitney $\mathrm{U}$ test and ${ }^{\mathrm{c}}$ the Kruskal-Wallis $\mathrm{H}$ test were used. ${ }^{\mathrm{d}}$ Statistically significant.

pcDNA4/myc-His C-H-Ras constructs were lysed with lysis buffer $(10 \mathrm{mM}$ Tris- $\mathrm{HCl} \mathrm{pH}$ 7.5, $150 \mathrm{mM} \mathrm{NaCl}, 1 \%$ Triton $\mathrm{X}-100$ and $1 \mathrm{mM}$ EDTA) containing protease inhibitor (1:25; Complete Protease Inhibitor Cocktail Tablets in glass vials, Roche) at $48 \mathrm{~h}$ after transfection. Cytosolic protein $(30 \mu \mathrm{g})$ was quantified by Bradford assay, separated by $12 \%$ SDSPAGE and transferred onto a PVDF membrane (Millipore). Then, the membrane was probed with monoclonal antibody against the myc tag (2 $\mu \mathrm{g} / \mathrm{ml}$; clone 9B11; Cell Signaling) and incubated in a peroxidase conjugated goat anti-mouse antibody (1 $\mu \mathrm{g} / \mathrm{ml}$; Rockland). SuperSignal West Dura Extended Duration Substrate (Pierce) was used for signal detection. For the $\beta$-actin signal, the blot was re-probed with $1.25 \mu \mathrm{g} / \mathrm{ml}$ monoclonal anti-actin $(2 \mu \mathrm{g} / \mathrm{ml}$; clone $\mathrm{C} 7$; Chemicon).

Focus formation assay. NIH3T3 cells were transfected by Lipofectamine $^{\mathrm{TM}} 2000$ (Invitrogen) according to the manufacturer's instructions. Twenty-four hours after transient transfection by a $4.0 \mu \mathrm{g}$ control (pcDNA4/myc-His C) or recombinant (pcDNA4/myc-His C-EVL and pcDNA4/
myc-His C-H-Ras) plasmids, identical numbers of cells were respectively plated into $35-\mathrm{mm}$ plates containing growth medium. Three replicate dishes were plated for each sample. After ten days of incubation, transformed foci were visualized by crystal violet staining.

Cell migration assay. A Boyden chamber assay was used with minor modification. Briefly, MCF-7 cells transfected with pEGFP-C1 or pEGFP-C1-EVL were used after $30 \mathrm{~h}$ of transient transfection. Viable cells $\left(2 \times 10^{4}\right)$ in $0.3 \mathrm{ml}$ DMEM containing $1 \%$ fetal bovine serum were seeded into the upper chamber of each Millicell-PCF insert ( $8 \mu \mathrm{m}$ pore size, polycarbonate filter, $12 \mathrm{~mm}$ diameter; Millipore) and the units were placed into 24-well plates containing DMEM supplemented with $10 \%$ fetal bovine serum. After $18 \mathrm{~h}$, cells remaining on the upper side of the filters were removed carefully with cotton swabs and the migratory cells on the membrane underside were identified by EGFP fluorescence. Six random microscope fields (magnification, x100) were counted in each replicate filter and results were expressed as the mean \pm SD. 


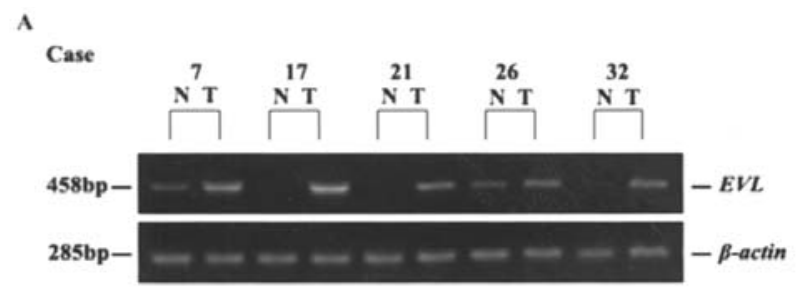

B

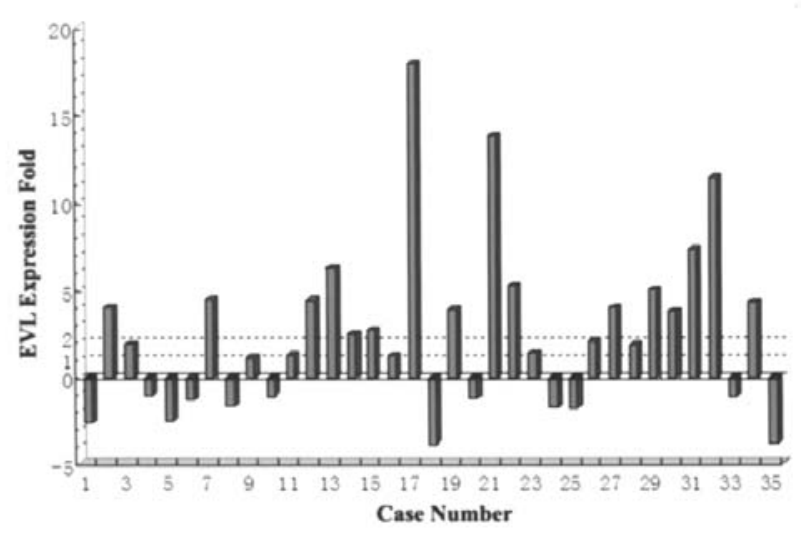

Figure 1. EVL mRNA expression in breast carcinomas. (A) Semi-quantitative RT-PCR analysis of EVL mRNA levels in five representative pairs of breast specimens. EVL mRNA was highly expressed in the tumor (T) samples although was either down-regulated or absent in normal breast specimens $(\mathrm{N})$. $\beta$-actin mRNA levels were used as an internal control. (B) Real-time RT-PCR analysis of $E V L$ expression in breast cancer specimens. Numbers indicate case numbers. Expression fold is the ratio of relative $E V L$ expression levels in normal and cancer tissues. A positive value indicated a higher expression level; a negative value indicated a lower expression level in tumors relative to the normal specimens. All data were averages of triplicate experiments.

Statistical analysis. SPSS 12.0 (SPSS, USA) for Windows was used for statistical analysis. A paired sample t-test was used to compare the relative $E V L$ expression levels between normal paired and tumor specimens. The Mann-Whitney $U$ test and the Kruskal-Wallis $\mathrm{H}$ test were used to test the association between the fold difference of $E V L$ transcripts in paired specimens and clinicopathological data. An independent sample t-test was used to compare variables in the cell migration assay. A P-value of $<0.05$ was considered to be statistically significant.

\section{Results}

EVL transcription levels are elevated in human breast cancer. Semi-quantitative RT-PCR was utilized to obtain an overview of the $E V L$ expression pattern in clinical breast cancer samples. Up-regulated $E V L$ mRNA expression was apparent in 20 out of $35(57.1 \%)$ breast tumors when it was compared to the normal tissue. Five representative cases are shown in Fig. 1A.

The quantification of $E V L$ mRNA was performed by using real-time RT-PCR. The result was normalized to the $\beta$-actin mRNA level of each specimen. As shown in Fig. 2, the relative expression levels of $E V L$ ranged from 0.00203 to 0.454 (median, 0.0396; mean, 0.0586) in normal breast tissues and ranged from 0.00814 to 0.567 (median, 0.0631 ; mean, 0.115) in breast cancer specimens. EVL expression levels in tumors were significantly higher than that of the matched normal tissues $(\mathrm{P}=0.032)$.

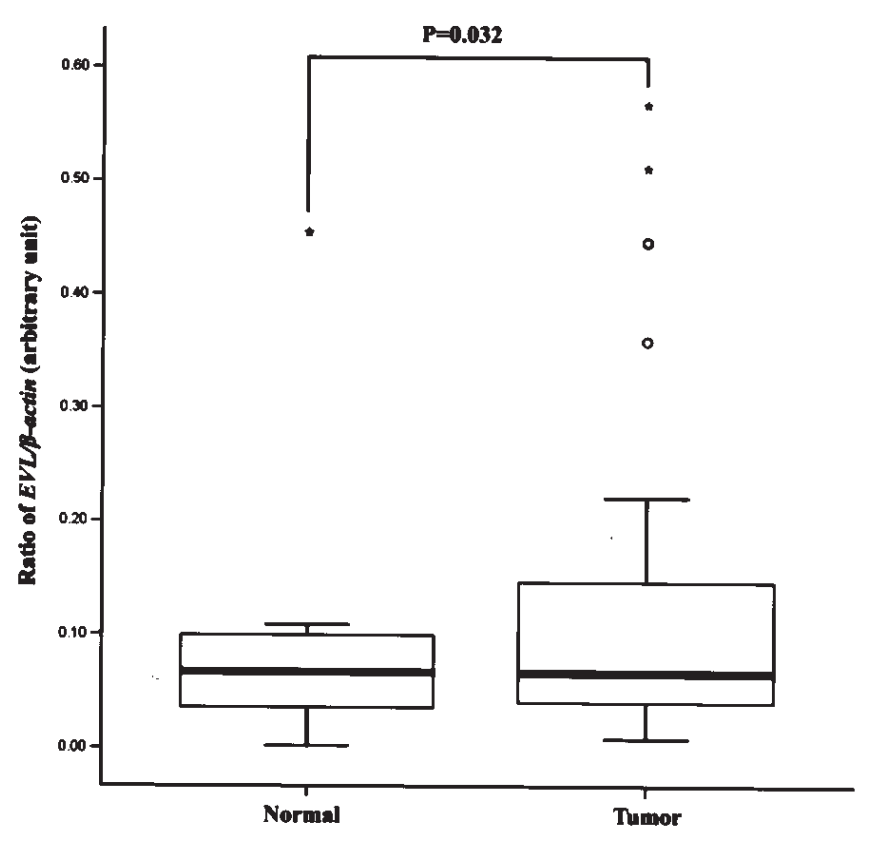

Figure 2. Comparison of the relative expression levels of $E V L$ in 35 paired normal breast and cancerous tissues. There was a significant increase in the mRNA expression of $E V L$ in tumors as compared to matched normal specimens ( $\mathrm{P}=0.032$, paired sample t-test). ${ }^{\circ}$ Mild outlier. ${ }^{*}$ Extreme outlier.

Up-regulated expression of EVL in breast cancer is correlated with clinical stages. To examine the difference in $E V L$ mRNA expression between the paired tumor and normal breast tissue, we compared the EVL/ $\beta$-actin mRNA ratios of the tumors to the ratios of the matched normal tissues. The fold differences are shown in Fig. 1B, which indicated that $65.7 \%$ (23 out of 35) of the tumors possessed elevated EVL mRNA expression and $48.6 \%$ (17 out of 35 ) of the tumors had more than a two-fold increase in EVL mRNA expression. Elevated expression levels of $E V L$ were observed in $40 \%$ (4 out of 10) of stage I, in $72.7 \%$ (16 out of 22 ) of stage II and in $100 \%$ ( 3 out of 3 ) of stage III tumors tested. The fold differences of paired specimens significantly correlate with clinical stages $(\mathrm{P}=0.021)$. No significant correlation, however, was found between the fold differences and tumor size, age, histology, lymph node status, ER status or PR status (Table I).

Focus formation ability of NIH3T3 is unaffected by EVL overexpression. The up-regulation of $E V L$ mRNA expression in a large fraction of breast cancer specimens and the implicated tumorigenesis ability of VASP (11) might suggest a role for $E V L$ in promoting transformation/tumorigenesis. To test this hypothesis, we transfected a mammalian expression construct with no insert (pcDNA4/myc-His C) or expressing the $E V L$ or $H$-Ras cDNA (pcDNA4/myc-His C-EVL and pcDNA4/myc-His C-H-Ras, respectively) into NIH3T3 cells and assessed focus formation after ten days of incubation. As shown in Fig. 3, there were no foci observed in the cells expressing EVL and the control pcDNA4/myc-His C-transfected cells. In contrast, oncogenic H-Ras expression effectively induced the foci formation of NIH3T3 cells. The result indicated that EVL overexpression was insufficient to induce NIH3T3 cell transformation in vitro. 

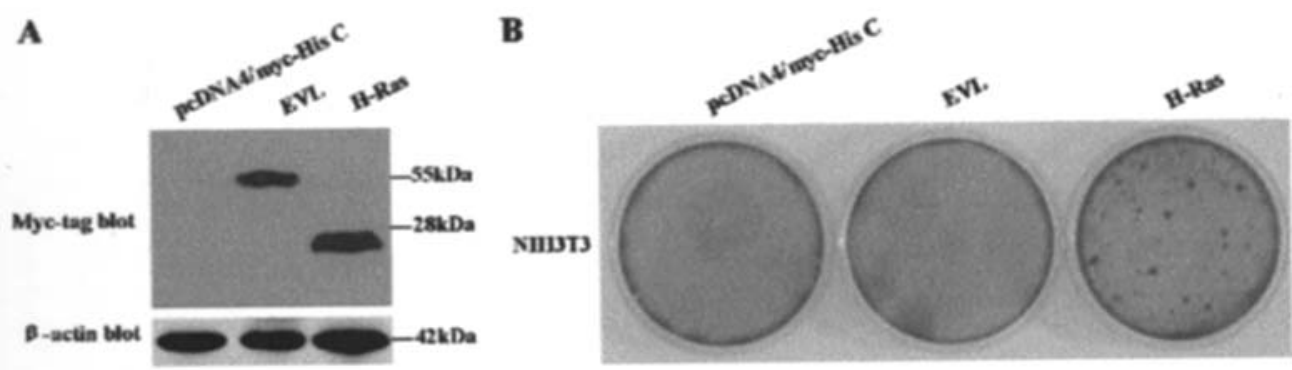

Figure 3. Transformation ability of EVL. NIH3T3 cells were transfected with pcDNA4/myc-His C, pcDNA4/myc-His C-EVL (EVL) or pcDNA4/myc-His C-H-Ras (H-Ras), respectively. (A) Western blot analysis of EVL and H-Ras protein expression in transient transfected NIH3T3 cells using an anti-c-myc tag monoclonal antibody. B-actin was the internal control for protein loading. (B) Focus formation assay, where NIH3T3 cells were transfected with the indicated expression plasmids and foci were visualized after 10 days by staining with crystal violet.
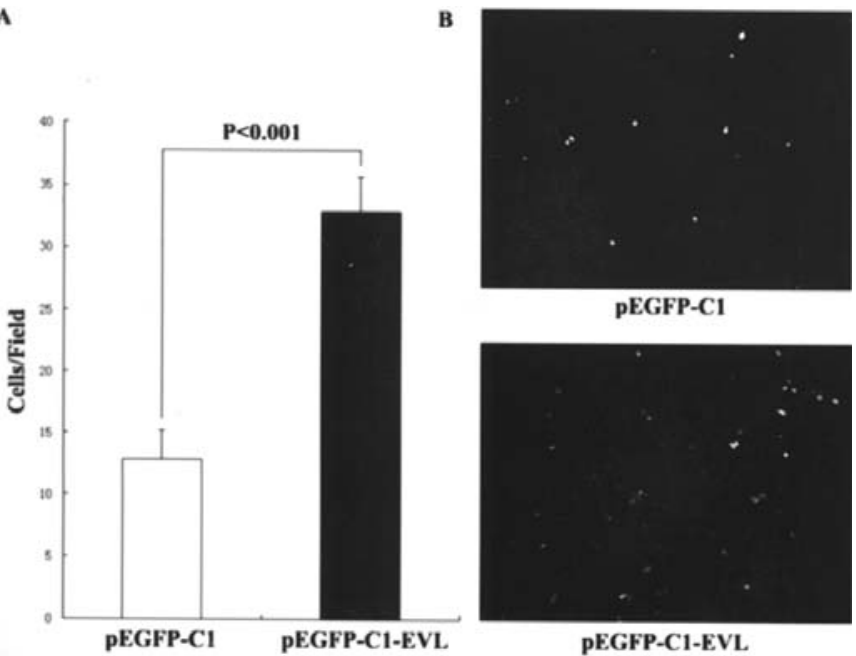

PEGFP-C1-EVI

Figure 4. Effect of transient EVL expression on the migration of MCF-7 cells. (A) Transient EVL expression significantly promoted MCF-7 cell migration as compared with the control $(\mathrm{P}<0.001$, independent sample t-test). Modified Boyden chamber migration assays were performed as described in Materials and methods. Data represent the mean \pm SD of three independent experiments. (B) Representative photographs of a migration assay for migrated MCF-7 cells expressing EGFP or EGFP-EVL (original magnification, x100).

Transient EVL expression promotes the migration of MCF-7 cells. Ena/VASP proteins are known to participate in signal pathways that affect cell adhesion and motility, which are the key components of invasion and metastasis. To clarify whether up-regulated EVL expression influences cancer cell migration, MCF-7 cells were transfected with an expression vector encoding EGFP (pEGFP-C1) or an EGFP fusion protein of EVL (pEGFP-C1-EVL) and examined in a modified Boyden chamber assay. As shown in Fig. 4, EVL-expressing cells exhibited an approximately three-fold increase in serumstimulated cell migration compared with control cells $(12.8 \pm 2.32$ versus $32.8 \pm 2.79 ; \mathrm{P}<0.001)$, which suggested that EVL positively regulated the motility of MCF-7 cells.

\section{Discussion}

Proteins of the Ena/VASP family share a conserved domain organization: a central proline-rich domain flanked by two defined regions called Ena/VASP homology domains (EVH1 and EVH2). They have emerged as multifunctional regulators of the actin cytoskeleton $(6,7)$. In human cancer, the upregulation of hMena and VASP has been observed via immunohistochemistry in the progression of carcinogenesis, though the underlying molecular mechanism remains to be elucidated $(8,9,11)$.

In human glioblastoma multiforme, elevated expression levels of $E V L$ in tumor tissues have been examined by microarray analysis (16). We utilized the SAGE (Serial Analysis of Gene Expression) databases of the CGAP (Cancer Genome Anatomy Project) to analyse the expression pattern of the $E V L$ gene in normal and cancerous breast tissues and cell lines by the analytical tool DGED (Digital Gene Expression Display), a four-fold up-regulation of EVL in cancerous tissues and cell lines was found (data not shown). In this report, we compared EVL mRNA expression levels in breast tumors with corresponding normal tissues originating from the same patient in order to determine whether it is involved in human breast cancer progression. By semiquantitative RT-PCR, $57.1 \%$ of breast tumors were shown to have exhibited up-regulation of $E V L$ expression. Increased $E V L$ expression level in breast cancer was further detected by more-sensitive real-time quantitative PCR, which showed that elevated $E V L$ expression was apparent in $65.7 \%$ of the breast cancer tissues. Although only $48.6 \%$ of the cases showed more than a two-fold elevated mRNA expression in the tumor, the observed up-regulation of $E V L$ expression in the tumor was statistically significant.

To evaluate the functional significance of the up-regulation of EVL expression in breast cancer, we examined the transforming properties of human $E V L$ by focus formation assay. However, the results demonstrated that $E V L$ expression was insufficient to induce NIH3T3 cell transformation in vitro.

To explore the role of EVL expression in the process of human breast cancer, we examined the correlation of $E V L$ mRNA expression level with the clinical features. As shown in Table I, the data demonstrated that increased EVL mRNA expression paralleled the advanced clinical stages. Notably, the increasing expression of VASP paralleled the clinical stages of lung adenocarcinomas (11) and the hMena protein was also reported to be progressively overexpressed in high-risk benign breast lesions $(8,9)$.

Active aberrant cell migration is a typical characteristic of the advanced stages of cancer. Ena/VASP proteins are known to act at the interface of several signaling pathways in order to regulate cellular protrusion and migration by controlling the 
geometry of growing actin networks. In this study, we found that overexpression of EVL can promote MCF-7 cell motility, which is an important step in the metastatic cascade. In epithelial cells, EVL is co-localized with the proteins which are highly implicated in the supramolecular complexes necessary for the establishment of cell-cell contacts (adherens junctions and tight junctions), though the role of EVL in this location is unclear (17). Additionally, the overexpression of a fragment of Ena/VASP responsible for oligomerization disrupts epithelial sheet formation (18). Since cell motility responses are dependent on a balance of cell contact formation and turnover (19), the promoted EVLoverexpressed MCF-7 cell motility may be due to the actions of EVL in regulating cell adhesion.

In conclusion, $E V L$ expression levels were higher in breast cancer tissues than in normal tissues. Furthermore, there was a statistically significant correlation between $E V L$ relative expression levels and clinical stages. Our current results suggest that overexpression of EVL may be an oncogenic event that is involved in the processes of malignancy, such as tumor invasion and/or metastasis.

\section{Acknowledgements}

We thank the doctors (Zheng-Jun Xi, et al) in the Pathological Division of Xinhua Hospital for their cooperation in the sample collection and pathological examination.

\section{References}

1. Parkin DM, Bray F, Ferlay J and Pisani P: Global cancer statistics, 2002. CA Cancer J Clin 55: 74-108, 2005.

2. Jin F, Devesa SS, Chow WH, Zheng W, Ji BT, Fraumeni JF and Gao YT: Cancer incidence trends in urban Shanghai, 1972-1994: an update. Int J Cancer 83: 435-440, 1999.

3. Yang L, Parkin DM, Ferlay J, Li L and Chen Y: Estimates of cancer incidence in China for 2000 and projections for 2005. Cancer Epidemiol Biomarkers Prev 14: 243-250, 2005.

4. Lambrechts A, Van Troys M and Ampe C: The actin cytoskeleton in normal and pathological cell motility. Int J Biochem Cell Biol 36: 1890-1909, 2004.

5. Wulfkuhle JD, Sgroi DC, Krutzsch H, McLean K, McGarvey K, Knowlton M, Chen S, Shu H, Sahin A, Kurek R, Wallwiener D, Merino MJ, Petricoin EF, Zhao Y and Steeg PS: Proteomics of human breast ductal carcinoma in situ. Cancer Res 62: 6740-6749, 2002.

6. Krause M, Dent EW, Bear JE, Loureiro JJ and Gertler FB: Ena/ VASP proteins: regulators of the actin cytoskeleton and cell migration. Annu Rev Cell Dev Biol 19: 541-564, 2003.
7. Kwiatkowski AV, Gertler FB and Loureiro JJ: Function and regulation of Ena/VASP proteins. Trends Cell Biol 13: 386-392, 2003.

8. Di Modugno F, Bronzi G, Scanlan MJ, Del Bello D, Cascioli S, Venturo I, Botti C, Nicotra MR, Mottolese M, Natali PG, Santoni A, Jager E and Nisticò P: Human Mena protein, a SEREX-defined antigen overexpressed in breast cancer eliciting both humoral and $\mathrm{CD} 8^{+} \mathrm{T}$-cell immune response. Int $\mathrm{J}$ Cancer 109: 909-918, 2004.

9. Di Modugno F, Mottolese M, Di Benedetto A, Conidi A, Novelli F, Perracchio L, Venturo I, Botti C, Jager E, Santoni A, Natali $\mathrm{PG}$ and Nisticò $\mathrm{P}$ : The cytoskeleton regulatory protein hMena (ENAH) is overexpressed in human benign breast lesions with high risk of transformation and human epidermal growth factor receptor-2-positive/hormonal receptor-negative tumors. Clin Cancer Res 12: 1470-1478, 2006.

10. Wang W, Goswami S, Lapidus K, Wells AL, Wyckoff JB, Sahai E, Singer RH, Segall JE and Condeelis JS: Identification and testing of a gene expression signature of invasive carcinoma cells within primary mammary tumors. Cancer Res 64: 8585-8594, 2004.

11. Dertsiz L, Ozbilim G, Kayisli Y, Gokhan GA, Demircan A and Kayisli UA: Differential expression of VASP in normal lung tissue and lung adenocarcinomas. Thorax 60: 576-581, 2005.

12. Gertler FB, Niebuhr K, Reinhard M, Wehland J and Soriano P: Mena, a relative of VASP and Drosophila Enabled, is implicated in the control of microfilament dynamics. Cell 87: 227-239, 1996.

13. Lambrechts A, Kwiatkowski AV, Lanier LM, Bear JE, Vandekerckhove J, Ampe C and Gertler FB: cAMP-dependent protein kinase phosphorylation of EVL, a Mena/VASP relative, regulates its interaction with actin and $\mathrm{SH} 3$ domains. J Biol Chem 275: 36143-36151, 2000.

14. Liu K, Li L, Nisson PE, Gruber C, Jessee J and Cohen SN: Reversible tumorigenesis induced by deficiency of vasodilatorstimulated phosphoprotein. Mol Cell Biol 19: 3696-3703, 1999.

15. Singletary SE, Allred C, Ashley P, Bassett LW, Berry D, Bland KI, Borgen PI, Clark G, Edge SB, Hayes DF, Hughes LL, Hutter RVP, Morrow M, Page DL, Recht A, Theriault RL, Thor A, Weaver DL, Wieand HS and Greene FL: Revision of the American Joint Committee on cancer staging system for breast cancer. J Clin Oncol 20: 3628-3636, 2002.

16. Fathallah-Shaykh HM, Rigen M, Zhao LJ, Bansal K, He B, Engelhard HH, Cerullo L, Roenn KV, Byrne R, Munoz L, Rosseau GL, Glick R, Lichtor T and DiSavino E: Mathematical modeling of noise and discovery of genetic expression classes in gliomas. Oncogene 21: 7164-7174, 2002.

17. Bournier O, Kroviarski Y, Rotter B, Nicolas G, Lecomte MC and Dhermy D: Spectrin interacts with EVL (Enabled/vasodilatorstimulated phosphoprotein-like protein), a protein involved in actin polymerization. Biol Cell 98: 279-293, 2006.

18. Vasioukhin V, Bauer C, Yin M and Fuchs E: Directed actin polymerization is the driving force for epithelial cell-cell adhesion. Cell 100: 209-219, 2000.

19. Lauffenburger DA and Horwitz AF: Cell migration: a physically integrated molecular process. Cell 84: 359-369, 1996. 